\title{
Severe hypothyroidism presenting as arthropathy of the fingers: a new case report
}

\begin{abstract}
We describe the case of a patient presenting with painful arthropathy of the distal interphalangeal joints of the fingers. She was initially believed to have chronic inflammatory rheumatism. High thyroid stimulating hormone values were discovered leading to the diagnosis of primary severe hypothyroidism. Rapid response of articular manifestations to thyroid hormone substitution suggests that hypothyroidism could be the cause of this articular impairment.
\end{abstract}

Keywords: hypothyroidism, arthropathy, bone, joint
Volume II Issue 6 - 2019

\author{
Hana Zoubeidi, Fatma Daoud, Imène Rachdi, \\ Ibrahim Arbaoui, Mehdi Somai, Zohra Aydi, \\ Besma Ben Dhaou, Fatma Boussema \\ Department of Internal medicine, Habib Thameur Hospital, Tunis \\ El Manar University, Tunisia
}

\author{
Correspondence: Imène Rachdi, Department of Internal \\ medicine, Habib Thameur Hospital, Tunis El Manar University, Ali \\ Ben Ayed Street, 1089 Tunis, Tunisia, \\ Email rachdi.imnel4@gmail.com
}

Received: October 25, 2019 | Published: December 19, 2019
Abbreviations: DIP, distal interphalangeal; anti-CCPs, antibodies against cyclic citrullinated peptides; $\mathrm{LDH}$, lactate dehydrogenase; $\mathrm{CPK}$, creatine phosphokinase

\section{Introduction}

Thyroid dysfunction may present with musculoskeletal signs and symptoms. ${ }^{1}$ Rheumatic complains during hypothyroidism were first described by many authors and included several manifestations mainly, chronic joint effusions; ${ }^{2}$ muscular pain ${ }^{3}$ and proximal muscle weakness. ${ }^{4}$ We report a patient who suffered from a painful arthropathy of the distal interphalangeal joints of the fingers which was the first manifestation of a severe unknown hypothyroidism.

\section{Case report}

Mrs. EH, 32 years old, with no particular medical history, was hospitalized for exploration of a bilateral Raynaud's phenomenon of both hands with arthralgia of large and small joints for more than six months. She complained of asthenia of progressive aggravation without other associated signs. The physical examination found a puffy face (Figure 1), cutaneous xerosis and sclerosis, a psychomotor slowing with a rasping voice. Osteoarticular examination showed bilateral and symmetrical edema of the fingers (Figure 2) and toes (Figure 3) with swelling and reducible subluxation of the distal interphalangeal (DIP) joints (Figure 4). The other joints of the hand were simply swollen on examination. The large joints were normal. The rest of the somatic examination was normal. In biology, the blood count showed normochromic normocytic anemia at $10.8 \mathrm{~g} / \mathrm{dl}$, a normal rate of leukocytes with a normal platelet count. The inflammation parameters were normal with a C-reactive protein at $6 \mathrm{mg} / \mathrm{l}$ and a sedimentation rate at $10 \mathrm{~mm} \mathrm{H1}$. Renal function was normal. Antinuclear antibodies, antibodies against cyclic citrullinated peptides (anti-CCPs) and rheumatoid factor were negative. Capillaroscopy showed normal capillary loops. X-rays of the hands (Figure 5) and forefeet (Figure 6) showed subcutaneous soft tissue swelling with joint space narrowing in the proximal interphalangeal without erosive carpitis or geodes. Faced with asthenia and psychomotor slowing, thyroid function tests showed a TSH at $750 \mu u n i t s / \mathrm{ml}(\mathrm{N}: 0.5-7)$. The diagnosis of severe peripheral hypothyroidism was retained and comforted by biological rhabdomyolysis with creatine phosphokinase (CPK) at $1749 \mathrm{IU} / 1$ (N: 38-171) and lactate dehydrogenase (LDH) at 593IU/1 $(\mathrm{N}<248)$. Cardiac echocardiography showed good segmental and global left ventricular function with circumferential pericardial effusion. Cervical ultrasound revealed a multinodular normal size thyroid gland with TIRADS II nodules. Antithyroglobulin and antiperoxydase antibodies were negative. The patient was treated with L-thyroxine with progressive doses. The evolution was marked by a clear clinical improvement with disappearance of arthralgia, regression of edema and persistence of a slight subluxation of the IPD without local inflammatory signs. Clinical and biological euthyroidism was perfectly obtained at 4 months of follow-up while the patient was under $100 \mu \mathrm{g} /$ day of L-thyroxine.

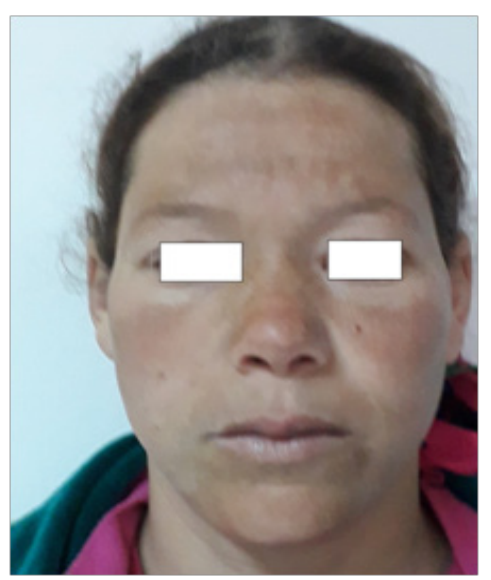

Figure I Puffy face with cutaneous xerosis.

\section{Discussion}

Disorders of the thyroid gland often present with musculoskeletal signs and symptoms. Conversely, rheumatic diseases, such as rheumatoid arthritis, Sjogren's syndrome and systemic lupus erythematosus, are frequently associated with autoimmune thyroid 
disease. ${ }^{1}$ None of these rheumatic diseases were present in this patient. Systemic sclerosis was initially suspected but early ruled out by the normality of complementary investigations. Several musculoskeletal symptoms have been reported in hypothyroidism but they are rarely the first manifestation of this endocrinopathy. Calcium pyrophosphate deposition disease was described in myxedematous patients who were symptomatic of joint pain in the hands and knees. ${ }^{5}$ The mechanism of this disorder is not well understood.

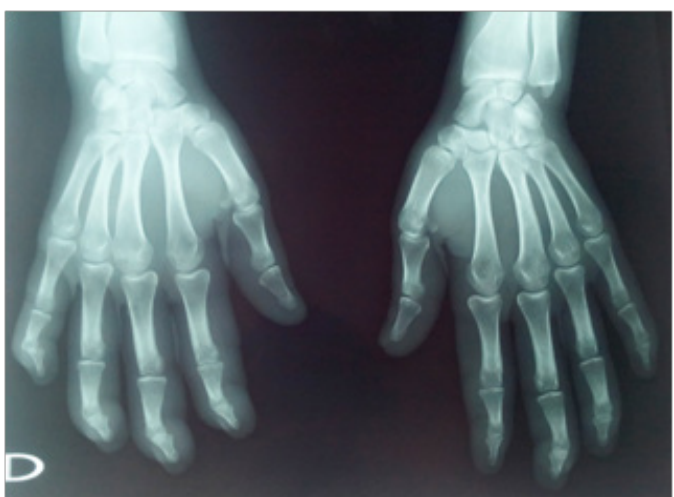

Figure 2 Edema of the fingers.

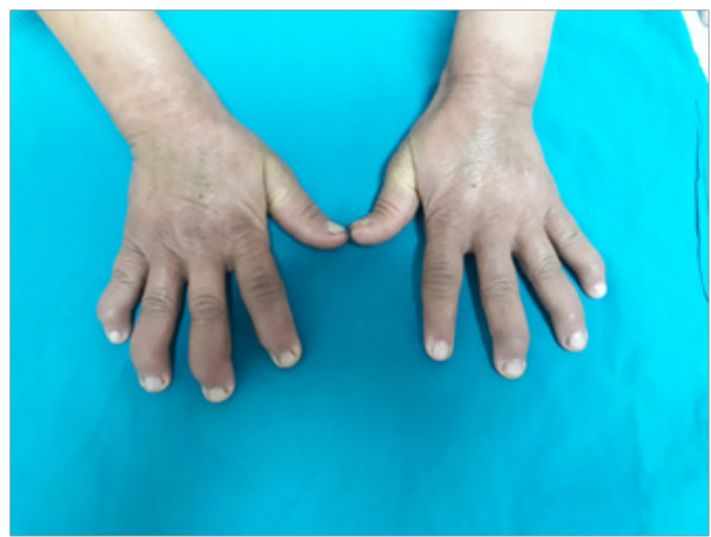

Figure 3 Edema of the toes.

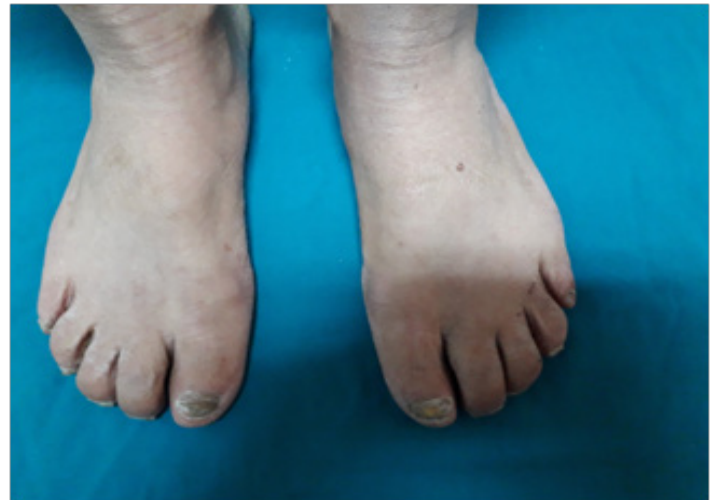

Figure 4 Swelling and reducible subluxation of the distal interphalangeal (DIP) joints.

Hypothyroid myopathy is also frequent. The symptoms often reported by patients are pain, cramps, stiffness, easy fatigability, and weakness. ${ }^{6,7}$ Our patient had asthenia without evidence of proximal muscle weakness neither muscle hypertrophy.

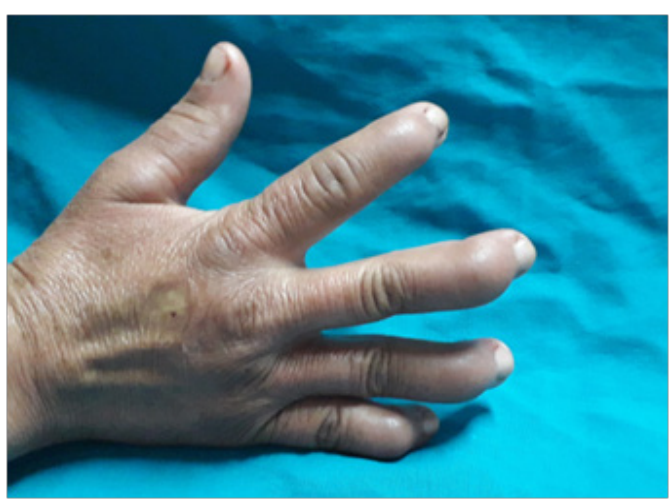

Figure 5 X-Rays of the hands showing subcutaneous soft tissue swelling with joint space narrowing in the proximal interphalangeal.

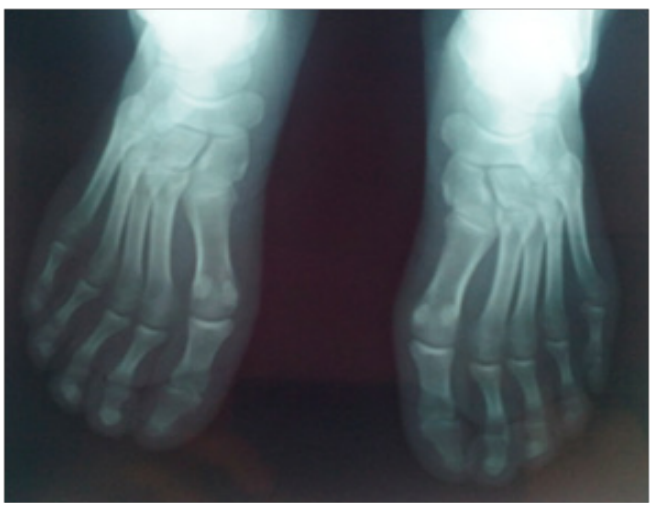

Figure 6 X-Rays of the forefeet showing subcutaneous soft tissue swelling with joint space narrowing in the proximal interphalangeal.

Serum muscle enzyme levels are frequently elevated in patients with hypothyroid myopathy and are elevated in up to $90 \%$ of asymptomatic patients. ${ }^{6}$ Myolysis was present in our patient despite the absence of evident muscle deficit.

Carpal tunnel syndrome is a common neuromuscular disorder that can be associated with hypothyroidism. ${ }^{8}$ In the present case, articular complaints were the presenting features of hypothyroidism. The articular involvement was mainly confined to the DIP joints. Clinical presentation was similar to erosive inflammatory rheumatism although the localization in the DIP is not common. Radiological images have not shown an evidence of bone or joint destruction, a difference from classical erosive arthritis.

Rheumatic complaints started simultaneously with the first symptoms of hypothyroidism. Our patient presented a rasping voice and a puffy face. The disappearance of joint pain and swelling, the clear improvement of deformation with thyroxine substitution and the absence of any evident underlying rheumatic disease suggest that hypothyroidism was responsible for the arthropathy in our patient.

\section{Conclusion}

The relationship between thyroid disorders and rheumatic manifestations is significant. Hypothyroidism could be a causative factor of joint complains. As the symptoms generally regress after substitutive treatment, as illustrated in our case, hypothyroidism should always be suspected in patients with rheumatic complaints of undeterminated etiology. 


\section{Acknowledgments}

None.

\section{Conflicts of interest}

The authors declare there are no conflicts of interest.

\section{References}

1. Anwar S, Gibofsky A. Musculoskeletal manifestations of thyroid disease. Rheum Dis Clin North Am. 2010;36(4):637-646.

2. Bland JH, Frymoyer JW. Rheumatic syndromes of myxedema. $N$ Engl J Med. 1970;282(21):1171-1174.

3. Wilke WS, Sheeler LR, Makarowski WS. Hypothyroidism with presenting symptoms of fibrositis. J Rheumatol. 1981;8(4):626-631.

4. Cabili S, Pines A, Kaplinsky N, et al. Hypothyroidism masquerading as polymyositis. Postgrad Med J. 1982;58(683):545-547.

5. Hartl E, Finsterer J, Grossegger C, et al. Relationship between thyroid function and skeletal muscle in subclinical and overt hypothyroidism. Endocrinologist. 2001;11(3):217-221.

6. Duyff RF, Van den Bosch J, Laman DM, et al. Neuromuscular findings in thyroid dysfunction: a prospective clinical and electrodiagnostic study. J Neurol Neurosurg Psychiatr. 2000;68(6):750-755.

7. Cruz MW, Tendrich M, Vaisman M, et al. Electroneuromyography and neuromuscular findings in 16 primary hypothyroidism patients. Arq Neuropsiquiatr. 1996;54(1):12-18.

8. Van Dijk MA, Reitsma JB, Fischer JC, et al. Indications for requesting laboratory tests for concurrent diseases in patients with carpal tunnel syndrome: a systematic review. Clin Chem. 2003;49(9):1437-1444. 